\title{
Magnetic vortex racetrack memory
}

\author{
Liwei D. Geng and Yongmei M. Jin* \\ Materials Science and Engineering Department, Michigan Technological University, \\ Houghton, MI 49931, USA
}

We report a new type of racetrack memory based on current-controlled movement of magnetic vortices in magnetic nanowires with rectangular cross-section and weak perpendicular anisotropy. Data are stored through the core polarity of vortices and each vortex carries a data bit. Besides high density, non-volatility, fast data access, and low power as offered by domain wall racetrack memory, magnetic vortex racetrack memory has additional advantages of no need for constrictions to define data bits, changeable information density, adjustable current magnitude for data propagation, and versatile means of ultrafast vortex core switching. By using micromagnetic simulations, current-controlled motion of magnetic vortices in cobalt nanowire is demonstrated for racetrack memory applications.

\section{Introduction}

Recent development of magnetic domain wall racetrack memory (DWRM) has received attention as an emerging non-volatile memory technology, promising high density, fast data access, and low power [1]. DWRM is based on current-controlled movement of domain walls in magnetic nanowires of in-plane $[1,2]$ or perpendicular $[3,4]$ anisotropy, where data are stored through the magnetization direction of magnetic domains in the nanowire. In DWRM, regularly spaced constrictions (or pinning sites) are fabricated along the racetrack to define the bit length and pin domain walls, and short current pulses are used to shift domain walls for data propagation. This paper presents a new racetrack memory, i.e., magnetic vortex racetrack memory (MVRM), using micromagnetic simulations. MVRM is based on current-controlled movement of magnetic vortices in magnetic nanowires of weak perpendicular anisotropy. In MVRM, data are stored through the vortex core polarity, and a series of data bits are encoded in an array of vortices in the nanowire. Unlike DWRM where the data bits are defined by the constrictions built in the nanowire, the data bits in MVRM are individual vortices that actually move in the nanowire under controlling current. This distinct feature of MVRM using mobile magnetic vortices as individual data carriers grants additional advantages over DWRM. In MVRM, since one vortex defines one data bit, there is no need for the regularly spaced constrictions along the racetrack, the information density (or the number of data bits) in a given racetrack can be varied by simply changing the number of magnetic vortices, and the magnitude of spin-polarized current for data propagation can be adjusted through vortex pile-up effect as will be explained. Moreover, MVRM can benefit from the ultrafast switching of magnetic vortex core polarity by versatile means, including pulsed magnetic field $[5,6]$, alternating current $[7,8]$, and circularly polarized light [9], which have been intensively investigated.

\footnotetext{
* Email: ymjin@mtu.edu

(C) 2016. This manuscript version is made available under the Elsevier user license http://www.elsevier.com/open-access/userlicense/1.0/
} 


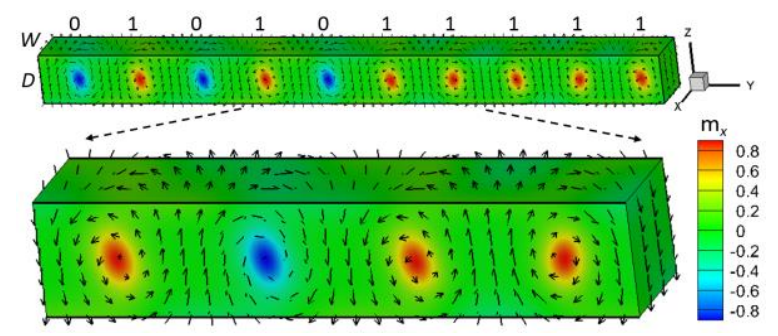

Figure 1. Micromagnetic simulation of vortices in Co nanowire. Data are stored through the core polarity of vortices. Arrows and color contours represent magnetization direction and its $x$ component (i.e., polarity), respectively.

An array of magnetic vortices can form in magnetic nanowires with weak perpendicular anisotropy as shown in Fig. 1, similar to the formation of well-known weak stripe domains in magnetic films of weak perpendicular anisotropy [10]. When the film thickness $D$ is below the critical thickness $D_{\text {cr }}$, the film is magnetized in the film plane complying with the strong demagnetization in the film normal direction; while above $D_{\text {cr }}$, so-called weak stripe domains form in the film with magnetization oscillating out of the film plane in a periodic manner to minimize the stray field energy [10]. Weak stripe domains take the shape of stripes (as so named) when viewed in the film plane while densely packed vortices (of a flux-closed character) when viewed in the film cross-section. Now consider a nanowire shown in Fig. 1 with the $z$-axis along the magnetic easy direction and the $y$-axis along the longitudinal direction of the wire. The magnetic domain structure in the nanowire resembles the weak stripe domains observed in films: magnetization oscillates up and down parallel to the magnetic easy direction ( $z$-axis) which forms an array of vortices in the $y z$-cross-section of the nanowire. If the core polarity $p$ is defined as 1 for pointing toward the positive and 0 for pointing toward the negative $x$-axis, the vortex array in the nanowire represents a series of data bits as exemplified in Fig. 1. It is worth noting one important difference in the vortex structure of nanowires from that of films. While vortices in films must be densely packed in a periodical manner [10], vortices in nanowires are in fact domain walls between neighboring magnetic domains thus can be placed with varied distances (see Fig. 6). In the nanowire, as compared to the film, demagnetization at the upper and lower $x y$-surface is reduced due to the small width in the $x$-direction, and additional stray field energy is generated at the front and back $y z$-surface because the magnetization at the vortex cores is perpendicular to them. Therefore, the periodic up and down oscillation of magnetization is no longer the unique configuration exclusively present in the film. In contrast, perpendicular domains of varied sizes form in the nanowire along the longitudinal direction, and the individual domain walls of a flux-closed character are the magnetic vortices that function as movable data bits in the nanowire. As shown in Fig. 3, the energy variation with the vortex separation distance (i.e., perpendicular domain size) is $\Delta F / \mu_{0} M_{s}^{2} V \sim 0.02$ and with the vortex core polarity distribution is $\Delta F / \mu_{0} M_{s}^{2} V \sim 0.002$, which is much smaller than the energy of weak anisotropy of the Co nanowire, $K_{\mathrm{u}} / \mu_{0} M_{s}^{2}=0.2$. As such, the number of vortices in a racetrack and the distance between them can be varied and the polarity of vortices can be switched, with insignificant 
changes in the system energy. This allows separate manipulation of individual vortices as required in data operation, by separating a specific vortex from its neighboring vortices into a designated place (e.g., the notch in Fig. 6) to be read or written (i.e., polarity switching) without interfering the polarity of neighboring vortices. This paper presents a micromagnetic simulation study of cobalt nanowires to demonstrate the current-controlled motion of magnetic vortices for racetrack memory applications.

\section{Micromagnetic modeling}

In micromagnetic modeling, magnetic domain structure is described by magnetization direction unit vector field $\mathbf{m}(\mathbf{r})$, which is set to zero outside the magnetic nanowire. The magnetization time evolution under magnetic field and spin-polarized current is described by the modified Landau-Lifshitz-Gilbert equation [11]:

$$
\partial \mathbf{m} / \partial t=\gamma \mathbf{H}^{\mathrm{eff}} \times \mathbf{m}+\alpha \mathbf{m} \times \partial \mathbf{m} / \partial t-(\mathbf{u} \cdot \nabla) \mathbf{m}+\beta \mathbf{m} \times[(\mathbf{u} \cdot \nabla) \mathbf{m}],
$$

where $\gamma$ is the gyromagnetic ratio, $\alpha$ the damping parameter, $\beta$ the nonadiabatic spin-transfer torque coefficient, $\mathbf{H}^{\text {eff }}$ the effective magnetic field, and $\mathbf{u}$ the electron motion velocity vector which is proportional to the electrical current density. $\mathbf{H}^{\mathrm{eff}}$ is determined by the variational derivative of the free energy $F, \mathbf{H}^{\mathrm{eff}}=-\delta F / \delta \mathbf{m}$, and $F$ is a sum of magnetic anisotropy energy, exchange energy, magnetostatic energy, and external magnetic energy [12]:

$$
\begin{aligned}
F=\int K_{\mathrm{u}}\{1 & \left.-[\mathbf{t}(\mathbf{r}) \cdot \mathbf{m}(\mathbf{r})]^{2}\right\} d^{3} r+A \int|\operatorname{grad} \mathbf{m}(\mathbf{r})|^{2} d^{3} r \\
& +\frac{\mu_{0} M_{s}^{2}}{2} f \frac{d^{3} k}{(2 \pi)^{3}}|\mathbf{n} \cdot \tilde{\mathbf{m}}(\mathbf{k})|^{2}-\mu_{0} M_{s} \int \mathbf{H}^{\mathrm{ex}} \cdot \mathbf{m}(\mathbf{r}) d^{3} r,
\end{aligned}
$$

where $K_{\mathrm{u}}$ is the magnetic anisotropy constant, $\mathbf{t}(\mathbf{r})$ the magnetic easy direction, $A$ the exchange stiffness constant, $\mu_{0}$ the permeability of vacuum, $M_{\mathrm{s}}$ the saturation magnetization, $\mathbf{H}^{\mathrm{ex}}$ the external magnetic field, $f$ the principal value integral excluding the point $\mathbf{k}=\mathbf{0}$, $\tilde{\mathbf{m}}(\mathbf{k})=\int \mathbf{m}(\mathbf{r}) e^{-i \mathbf{k} \cdot \mathbf{r}} d^{3} r$, and $\mathbf{n}=\mathbf{k} / k$ [13]. Eq. (1) is numerically solved using our own Fortran 90 code with parallel algorithm by Message Passing Interface (MPI), which has been developed in our group and used in previous works $[12,14,15]$. The simulations are performed on Stampede supercomputer at Texas Advanced Computing Center for cobalt nanowires with the magnetic easy direction $\mathbf{t}(\mathbf{r})$ along the $z$-axis and the material parameters [16] $M_{\mathrm{s}}=1.4 \times 10^{6} \mathrm{~A} / \mathrm{m}$, $K_{\mathrm{u}}=5.2 \times 10^{5} \mathrm{~J} / \mathrm{m}^{3}$, and $A=3 \times 10^{-11} \mathrm{~J} / \mathrm{m}$. Based on the experimentally deduced damping parameter $\alpha$ in $\mathrm{Co}$ thin films $[17,18]$ and nonadiabatic spin-transfer torque coefficient $\beta$ in $\mathrm{Co} / \mathrm{Pt}$ multilayer nanowires $[19,20], \alpha=0.2$ and $\beta=0.4$ are used in our simulations. A $144 \times 144 \times 288$ computational cell with grid size of $2 \mathrm{~nm}$ is used. In cobalt, the typical magnetostatic interaction energy $K_{\mathrm{d}}=\mu_{0} M_{s}^{2} / 2=1.2 \times 10^{6} \mathrm{~J} / \mathrm{m}^{3}$, the reduced anisotropy $Q=K_{\mathrm{u}} / K_{\mathrm{d}}=0.42(<1$, thus is of weak perpendicular anisotropy), and the critical film thickness $D_{\mathrm{cr}}=30 \mathrm{~nm}$ (as obtained from Fig. 
3.109 in Ref. [10]). We choose a thickness of $D=48 \mathrm{~nm}$, greater than $D_{\text {cr }}$, to study magnetic vortex behaviors in nanowires.

\section{Results and discussion}

The effects of the nanowire shape on magnetic vortex structure are studied first. Three nanowires of $576 \mathrm{~nm}$ long but with different thickness $D$ and width $W$ are considered: $24 \mathrm{~nm} \times 48 \mathrm{~nm}, 48 \mathrm{~nm} \times 48 \mathrm{~nm}$, and $48 \mathrm{~nm} \times 24 \mathrm{~nm}$. Magnetic domain evolution is simulated starting from an initial configuration of uniform magnetization along the positive $x$-axis under random noise, assuming a periodic boundary condition along the $y$-direction. The simulated final domain structures for the three cases are shown in Fig. 2. For the small thickness $D=24 \mathrm{~nm}$ (below $D_{\text {cr }}$ ) in Fig. 2(a), the nanowire is magnetized uniformly in the $x y$-plane along the longitudinal direction, as expected. For the increased thickness $D=48 \mathrm{~nm}$ (above $D_{\text {cr }}$ ) in Fig. 2(b), an array of vortices form in the nanowire (similar to the weak stripe domains in magnetic films of weak perpendicular anisotropy), which separate alternating up and down magnetic domains. The magnetization at the vortex cores is along the positive $x$-axis as determined by the initial condition. Similar vortex structure forms when the thickness of the nanowire is kept the same while the width is reduced to $W=24 \mathrm{~nm}$ as shown in Fig. 2(c). However, the reduced width leads to a bigger separation distance between vortices thus a reduced number of vortices in the nanowire, i.e., $N=8$ for $W=24 \mathrm{~nm}$ in Fig. 2 (c) vs. $N=10$ for $W=48 \mathrm{~nm}$ in Fig. 2(b), where $N$ is the number of vortices in the $576 \mathrm{~nm}$ length wire. This is because a decrease in the width increases the demagnetization strength in the $x$-direction, which dictates a reduced number of vortices that generate stray field in the $x$-direction at their cores. When bringing two vortices closer, their interaction energy increases giving rise to a repulsive force between them, and there exists a critical distance below which two vortices would collapse in annihilation. The critical distance is a function of the wire shape, and determines the maximum number density of vortices in the nanowire. The simulations show that the maximum number of vortices allowed in the nanowire of $576 \mathrm{~nm}$ length is $N=12$ for $48 \mathrm{~nm} \times 48 \mathrm{~nm}$ cross-section while $N=10$ for $48 \mathrm{~nm} \times 24 \mathrm{~nm}$ crosssection. Next, the vortex interactions in the nanowire are investigated by studying the effects of vortex number density and polarity distribution on the system energy.

(a)

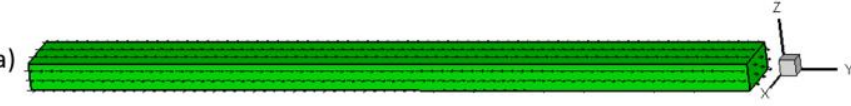

(b)

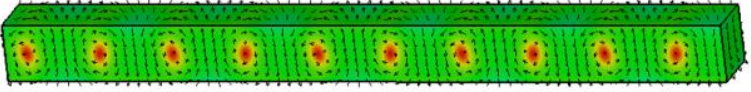

(c)

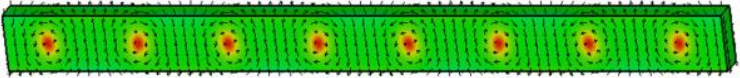

Figure 2. Nanowires of rectangular cross-section with thickness $\times$ width: (a) $24 \mathrm{~nm} \times 48 \mathrm{~nm}$, (b) $48 \mathrm{~nm} \times 48 \mathrm{~nm}$, and (c) $48 \mathrm{~nm} \times 24 \mathrm{~nm}$. 
The system energy in Eq. (2) is computed for a number of vortex configurations in the $576 \mathrm{~nm}$ long nanowire of $48 \mathrm{~nm} \times 48 \mathrm{~nm}$ cross-section in Fig. 2(b). The vortex configurations considered in the following simulations are obtained by relaxing predesigned magnetization distributions in the nanowire, unlike those in Fig. 2 developed from uniform magnetization under random noise. In practice, the vortex configurations representing any specific data structures are generated via data writing operation by a writing element integrated with the nanowire (racetrack). First, different numbers of evenly distributed vortices of the same polarity $p=1$ are considered. Due to the periodic boundary condition in the $y$-direction, the number of vortices can be varied by an increment of two. Figure 3(a) shows the system energy change as a function of the vortex number density, using the energy of uniform magnetization along the easy magnetic $z-$ direction as the reference. In the beginning, the energy reduces as the number of vortices increases due to a reduction in the stray field energy on the upper and lower surface. When the vortex number density reaches beyond $N=8$, the energy increases instead due to the increased repulsive interaction of vortices as they come too close. It is found that the nanowire cannot accommodate more than $N=12$ vortices per 576nm length. Next, for the given number density $N=10$, five more cases of different vortex core polarity distributions are considered, namely B-F as shown in Fig. 3(b), in addition to the case A of uniform polarity $p=1$. It is seen from the inset of the Fig. 3(a) that the energy is lower when neighboring vortices possess opposite core polarity, which is easy to understand from the viewpoint of core-core interaction: opposite polarity cores attract each other while same polarity cores repel through their magnetostatic interaction. Nevertheless, the energy change due to a change in vortex core polarity distribution is very small compared to that due to vortex number density, indicating a negligible contribution of vortex core polarity configuration to the interaction between vortices. Therefore, the vortex behaviors in the nanowire would be affected by the vortex number density (data density) while not by the vortex core polarity distribution (data information), and the former can be used as a control variable for adjusting the controlling current to move vortices in the nanowire, as will be discussed next in the simulation study of current-driven vortex motion.

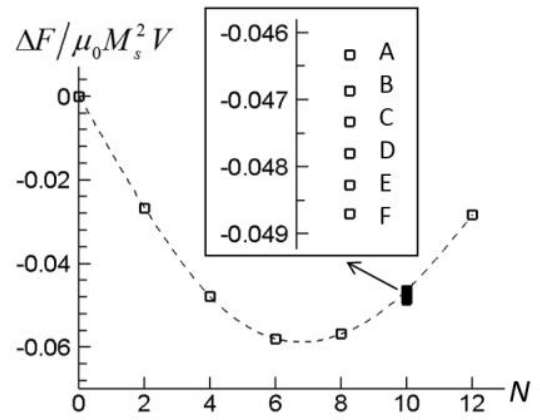

(a)

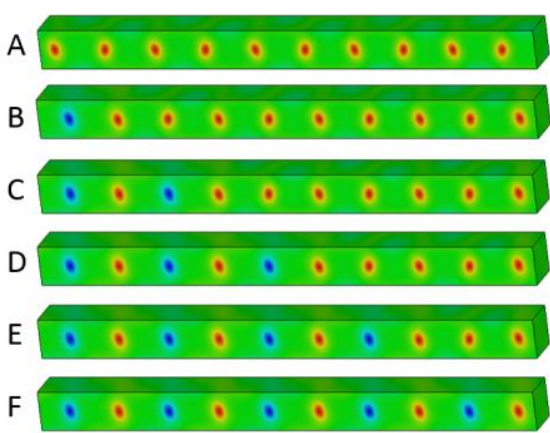

(b)

Figure 3. (a) System energy density as a function of the number density and the polarity distribution of magnetic vortices in the nanowire with $48 \mathrm{~nm} \times 48 \mathrm{~nm}$ square cross-section. Dashed line is guide to the eye. (b) Six different core polarity distributions A-F for 10 vortices $(N=10)$. 
The current driven vortex motion under constant current condition is simulated first in the nanowire of $48 \mathrm{~nm} \times 48 \mathrm{~nm}$ cross-section that is free of pinning sites. Two different current conditions, $u=20 \mathrm{~m} / \mathrm{s}$ and $u=100 \mathrm{~m} / \mathrm{s}$, are applied to a vortex array with number density $N=10$ in Fig. 4. Using the relation $u=J P g \mu_{B} / 2 e M_{s}$ between the electrical current density $J$ and the electron motion velocity $u$, and considering the polarization rate of the current $P=0.7$ [20], $u=20 \mathrm{~m} / \mathrm{s}$ corresponds to $J=0.69 \times 10^{8} \mathrm{~A} / \mathrm{cm}^{2}$. The simulation results show that the array of vortices move in the direction of electron flow (opposite to the direction of the current flow) as expected, at speed of $40 \mathrm{~m} / \mathrm{s}$ and $200 \mathrm{~m} / \mathrm{s}$, respectively. In both cases, the vortex motion velocity $v$ is related to the electron motion velocity $u$ by $v / u=\beta / \alpha$, same as the domain walls in Permalloy nanowires [11, $12,21]$. Moreover, changes in the vertical position of vortices under different currents are observed. In the two snapshots of the moving vortices in Fig. 4, vortices deviate away from the centerline position slightly under $u=20 \mathrm{~m} / \mathrm{s}$ while significantly under $u=100 \mathrm{~m} / \mathrm{s}$, where the deviation is to the lower edge for polarity $p=1$ while to the upper edge for polarity $p=0$ and the vortex shape becomes asymmetric when shifted to the edges. Such behaviors of vortices in Co nanowire is consistent with vortex domain wall behaviors in widely investigated Permalloy nanowires [21-23] while also exhibit some distinct features: the neighboring vortices possess opposite chirality, the vortices do not undergo the wall structure transformation from vortex to transverse, and the vortex size is much smaller here ( $40 \mathrm{~nm}$ vs. $\sim 300 \mathrm{~nm})$. These differences between Co nanowire and Permalloy nanowire are mainly due to the perpendicular magnetic anisotropy in the former in contract to the in-plane magnetic anisotropy in the latter. Nevertheless, vertical displacement of vortices is insignificant in Co nanowires in MVRM application because the current density required to move vortices is much lower than $100 \mathrm{~m} / \mathrm{s}$ due to the domain wall pile-up effect, as will be discussed in the following.

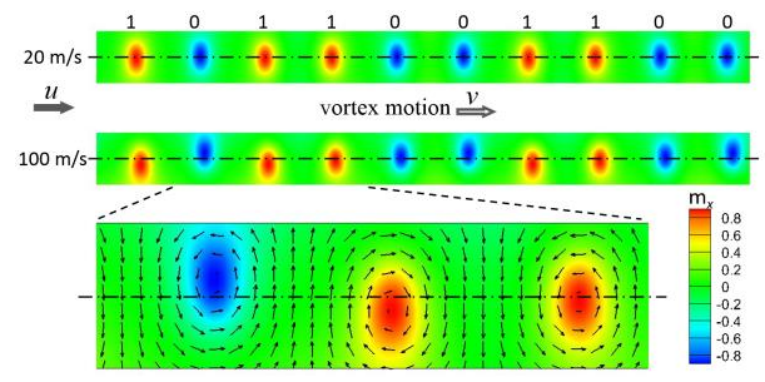

Figure 4. Simulated snapshots of moving vortex array under electrical current $u=20 \mathrm{~m} / \mathrm{s}$ and $u=100 \mathrm{~m} / \mathrm{s}$ in the central $y z$ plane of the nanowire with $48 \mathrm{~nm} \times 48 \mathrm{~nm}$ square cross-section. Black arrows and color contours represent magnetization direction and its $x$-component, respectively. The direction of electron flow $u$ is shown in solid gray arrow while the direction of vortex motion $v$ in hollow gray arrow.

An important issue of the current-driven vortex motion is to determine the critical depinning current for the pinning sites present in the nanowire. Although the constrictions are not needed to define the data bits in MVRM, some pinning sites are required to facilitate certain 
operations on the vortices, including writing and reading data. For example, in order to perform a writing operation on a vortex, pinning of the vortex is required to place the vortex in the designated position to undergo the writing operation (i.e., core polarity switching). Afterwards, depinning of the vortex is required to move the vortex out of the pinning site and move in another vortex for the next writing operation. Also, the number and spatial distribution of notches in the nanowire can serve as design parameters to define the controlling current for data operation in the racetrack, as will be discussed later. It should be noted that, in MVRM, only a few notches are required for the entire nanowire to facilitate certain data operations, while in DWRM, the entire nanowire is filled with densely packed notches since the number of notches is equal to the number of the data bits (each data bit is defined by one constriction). In our simulations, a pinning site made of square notch of $48 \mathrm{~nm} \times 48 \mathrm{~nm} \times d \mathrm{~nm}$ cut in both the front and back surfaces of the nanowire (type I notch) is considered, as shown in Fig. 5(a). A vortex would be held within the notch separated from other vortices for writing or reading operations. As a special case, the densely placed pinning sites in Fig. 5(b) resemble the constrictions in DWRM, where each pinning site holds one data bit. While switching the core polarity of a vortex at the pinning site is out of the scope of this paper, it is worth noting that ultrafast switching of magnetic vortex (via pulsed magnetic field, alternating current, and circularly polarized light) has been intensively investigated [5-9] and can be applied to MVRM for ultrafast data writing.

(a)

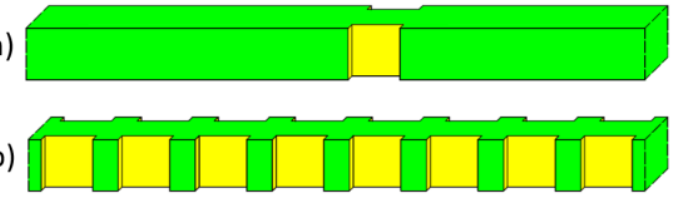

(b)

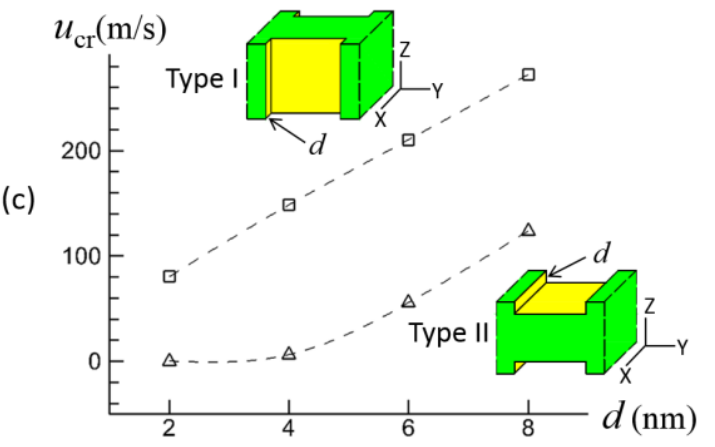

Figure 5. (a) One pinning site and (b) densely placed pinning sites made of square notch of $48 \mathrm{~nm} \times 48 \mathrm{~nm} \times d \mathrm{~nm}$ cut in the front and back surface of the nanowire. (b) Simulated critical depinning current (through electron motion velocity $u_{\mathrm{cr}}$ ) as a function of the notch depth $d$ for type I and type II notches. Dashed lines are guides to the eye.

The pinning strength of type I notch depends on the notch depth $d$. The critical depinning current, however, depends not only on the pinning strength of the notch, but also strongly on the number of vortices in the nanowire since they together generate domain wall pile-up effect which drastically amplifies the driving force to vortex motion [12]. To distinguish the intrinsic pinning 
strength of the notch from the extrinsic effect of the vortex number on the critical depinning current, the nanowire in Fig. 5(b) is considered where one vortex is located in the center of each notch as initial configuration. Since a current pulse is used to move data bits in the racetrack [14] as shown in Fig. 6, the critical depinning current for a given notch is determined under pulsed current condition. A series of current pulses each of $8 \mathrm{~ns}$ duration (exceeding the time required for a vortex to move out of the notch) with slowly increasing amplitude in steps of $6 \mathrm{~m} / \mathrm{s}$ is applied, where each current pulse starts with the same initial vortex configuration. When current is applied, the vortices move in the direction of electron motion away from their initial positions but soon get pinned at their individual notch boundary. The critical depinning current is determined by simulations, at which the vortices get depinned and move across the notch boundaries in the electron motion direction. It is shown in Fig. 5(c) that, when the notch depth increases the critical depinning current increases, indicating higher pinning strength as expected. As a comparison, a different pinning site with the square notch cut in the upper and lower surface of the nanowire (type II notch) is also considered in the simulations, and the critical depinning current as a function of the notch depth is obtained as shown in Fig. 5(c). It is seen that type I notch provides a stronger pinning strength than type II notch given the same notch depth. This is because type I notch removes the center part of the vortex with higher energy concentration while type II notch removes the boundary part of the vortex with lower energy concentration. Using the more effectively pining type I notch, current-driven vortex motion in the nanowire is simulated next. In particular, we choose $d=4 \mathrm{~nm}$ providing $u_{\mathrm{cr}}=148.5 \mathrm{~m} / \mathrm{s}$, and consider vortex arrays of different number densities.

We first consider $N=10$ shown in Fig. 6(a), where one vortex is trapped in the notch while the rest nine are placed in the notch-free part of the nanowire. Fig. 6(a) shows that a pulse of current with $u=20 \mathrm{~m} / \mathrm{s}$ and $t=3 \mathrm{~ns}$ duration pushes the trapped vortex out and moves in the next vortex, and a series of consecutive pulses repeat such process to shift the vortices one-by-one in sequence, realizing data propagation to the right along the nanowire. During the simulated vortex motion, as facilitated by the imposed periodic boundary condition, the number of vortices does not change on both sides of the notch, keeping the same number density of vortices in the nanowire. In a real nanowire in application, the number density of vortices can be controlled by two functional elements placed at the two ends of the nanowire that generate (feeder) and annihilate (receiver) one vortex under each current pulse. The current magnitude of $u=20 \mathrm{~m} / \mathrm{s}$ used here is significantly smaller than $u_{\mathrm{cr}}=148.5 \mathrm{~m} / \mathrm{s}$ that characterizes the pinning strength of the notch shown in Fig. 5(c). Such a drastic reduction in depinning current originates from the domain wall pile-up effect in the nanowire which is studied in detail in Ref. [12]: an array of domain walls stopped by a barrier in a magnetic nanowire form a domain wall pile-up which experiences wall-wall interaction under spin-polarized current; the wall-wall interaction gives rise to a driving force to the front domain wall in addition to its own current-induced driving force; and due to the contribution from the wall-wall interaction, the critical current to depin the front wall to pass the barrier is reduced by roughly a factor of $n$, the number of the domain walls 
in the pile-up. Such pile-up effect from the nine vortices in the nanowire behind the notch accounts for the drastically reduced current $u=20 \mathrm{~m} / \mathrm{s}$ as compared to $u_{\mathrm{cr}}=148.5 \mathrm{~m} / \mathrm{s}$ for no pile-up effect.

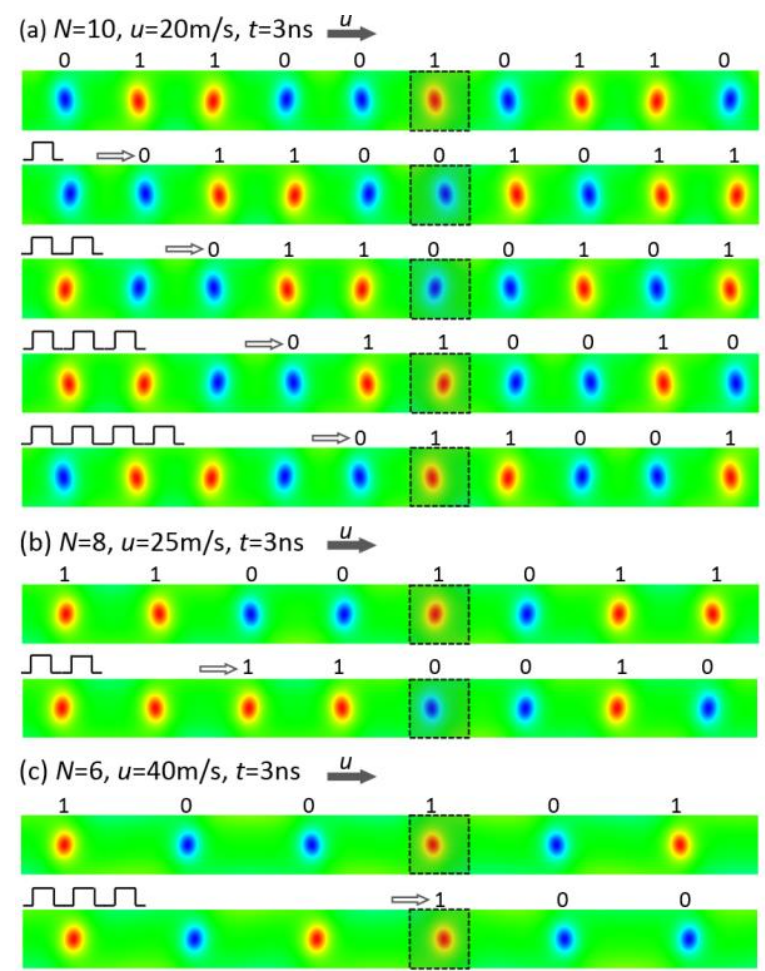

Figure 6. Simulated data propagation under pulsed current in $576 \mathrm{~nm}$-long nanowire of $48 \mathrm{~nm} \times 48 \mathrm{~nm}$ square cross-section with one $4 \mathrm{~nm}$-deep type I notch positioned at the shaded square enclosed in dashed line. (a) The sequential one-by-one shift of vortices under controlling current pulse with $u=20 \mathrm{~m} / \mathrm{s}$ and duration $3 \mathrm{~ns}$ for $N=10$. Increased current is required for reduced number density of vortices: (b) $u=25 \mathrm{~m} / \mathrm{s}$ for $N=8$ and (c) $u=40 \mathrm{~m} / \mathrm{s}$ for $N=6$. The direction of electron flow $u$ is shown in solid gray arrow while the direction of vortex motion in hollow gray arrow.

It is noted that, for a given current magnitude, the current pulse duration time must be matched to ensure the shift of only one vortex at a time. When the number of vortices behind the notch is changed, controlling current magnitude must be adjusted accordingly to shift the vortices in the nanowire. Two more different cases of vortex number density $N=8$ and $N=6$ are considered in the simulations, as shown in Figs. 6(b) and 6(c). The simulations show that, keeping the same pulse duration of $3 \mathrm{~ns}$, an increase of current pulse magnitude to $u=25 \mathrm{~m} / \mathrm{s}$ and to $u=40 \mathrm{~m} / \mathrm{s}$ leads to shifting of the vortex array of number density $N=8$ and $N=6$ in the nanowire by one vortex per current pulse respectively, in the same manner as in the case of $N=10$ in Fig. 6(a). The shifted vortex arrays after two consecutive pulses for $N=8$ and after three consecutive pulses for $N=6$ are exemplified in Fig. $6(\mathrm{~b})$ and (c). The range of current density and pulse duration required to move vortex array by one vortex for different number densities and notch 
varieties are being investigated and will be reported in a future work. Electron motion velocity $u$ of 20,25 , and $40 \mathrm{~m} / \mathrm{s}$ corresponds to current density $J$ of $0.69 \times 10^{8}, 0.86 \times 10^{8}$, and $1.38 \times 10^{8}$ $\mathrm{A} / \mathrm{cm}^{2}$, which are comparable to $\sim 10^{8} \mathrm{~A} / \mathrm{cm}^{2}$ used in domain wall racetrack memory nanowires $[1,3,4]$. It is worth noting that, according to the domain wall pile-up mechanism [12], an increase in the number of vortices ahead of the pinning site (by locating the pinning sites farther apart in the nanowire while keeping the same number density of vortices) would result in an inversely proportional reduction in the required current density, enabling current density significantly lower than $10^{8} \mathrm{~A} / \mathrm{cm}^{2}$ in MVRM applications. As such, the significant role of domain wall pile-up effect can be utilized in MVRM to effectively tailor the vortex motion behavior in the nanowire as driven by controlling current. The design of MVRM must take into account above discussed issues, including the nanowire cross-section geometry, the maximum number density of vortices, the type of pinning sites and their spatial distribution, and the current magnitude and pulse duration.

Finally, it is worth noting that the damping parameter $\alpha$ varies with the quality of the nanowire or film samples [18] thus it deserves to mention the effects of $\alpha$ on the above discussed vortex behaviors. Since $\alpha$ affects the dynamics of magnetic domain evolution but not the system free energy, a change in its value would not change the qualitative domain phenomena of vortex formation and current-driven vortex motion and depinning shown in Figs. 1-6 but only changes the quantitative values of the vortex motion velocity and the magnitude and duration of the controlling current pulses. In comparison with the above investigated case of $\alpha=0.2$, a significantly reduced value of $\alpha=0.04$ corresponding to another Co film in the experiments in Ref. 18 is also considered in the simulations. Given $\alpha=0.04$, the vortex motion velocity under electric current $u=20 \mathrm{~m} / \mathrm{s}$ in Fig. 4 becomes $200 \mathrm{~m} / \mathrm{s}$, the depinning current for notch depth $d=4 \mathrm{~nm}$ in Fig. 5 is $68 \mathrm{~m} / \mathrm{s}$, the duration and magnitude of the controlling current pulse are respectively $2 \mathrm{~ns}$ and $12 \mathrm{~m} / \mathrm{s}$ for sequential one-by-one shift of vortices in Fig. 6(a), while the vortex phenomena demonstrated for racetrack memory applications remain qualitatively the same as expected.

\section{Conclusions}

In summary, a new type of racetrack memory based on current-driven magnetic vortex motion in nanowires of weak perpendicular anisotropy is investigated. In particular, currentcontrolled motion of magnetic vortices in cobalt nanowire of rectangular cross-section is demonstrated using micromagnetic simulations and several influencing factors are addressed, including nanowire geometry, pinning sites, vortex number density, and vortex interactions. It is shown that magnetic vortices form when the nanowire thickness along magnetic easy direction is above the critical thickness $D_{\text {cr }}$ (similar to the weak stripe domains in magnetic films of weak perpendicular anisotropy), and that the minimum separation distance between vortices determining the maximum number density of vortices depends on the nanowire width (due to 
vortex interactions). The energy dependence on the number density of vortices and the vortex core polarity distribution is evaluated, and the vortex behaviors in Co nanowires under current are discussed in comparison with the domain wall behaviors in widely investigated Permalloy nanowires. The effects of pinning sites on vortex motion are also studied by considering square shape notches; pinning sites are required to perform certain data operations on vortices by writing and reading devices that are integrated elements of the racetrack. The pinning strength of a notch is examined with respect to the notch depth, and controlled motion of vortex arrays passing through the notch is simulated. It is shown that, due to the domain wall pile-up effect, the magnitude of controlling current significantly decreases as the number of vortices lined up behind the notch increases, which can be utilized to effectively tailor current-driven vortex motion behaviors in MVRM.

In MVRM, data are stored through the core polarity of vortices and are transported by vortices that move under controlling current. Thereby, the data bits are defined by individual movable vortices, in contrast to the data bits defined by built-in constrictions (i.e., notches) of the nanowire in DWRM. This leads to important distinct features of MVRM from recent development of domain wall racetrack memory [24], including no need for constrictions to define data bits, changeable data density, and adjustable current magnitude for data propagation. In addition, versatile means of ultrafast vortex switching [5-9] and vast knowledge from extensive investigations of magnetic vortices (see bibliography in Ref. [25]) in both scientific and technological communities may provide new opportunities to further develop magnetic vortex racetrack memory technology.

\section{Acknowledgements}

Support from NSF under Grant No. DMR-1409317 is acknowledged. Simulations were performed on XSEDE supercomputers.

\section{References}

[1] S. S. P. Parkin, M. Hayashi, L. Thomas, Science, 320, 190 (2008).

[2] A. J. Annunziata et al., Proc. of IEEE IEDM, 11-539 (2011).

[3] Y. Zhang, W. S. Zhao, D. Ravelosona, J. -O. Klein, J. V. Kim, C. Chappert, J. Appl. Phys., 111, 093925 (2012).

[4] Y. Zhang, W. S. Zhao, J. -O. Klein, C. Chappert, D. Ravelosona, J Phys. D: Appl. Phys., 48, 105001 (2015).

[5] B. Van Waeyenberge et al., Nature, 444, 461 (2006).

[6] R. Hertel, S. Gliga, M. Fähnle, C. M. Schneider, Phys. Rev. Lett., 98, 117201 (2007).

[7] K. Yamada, S. Kasai, Y. Nakatani, K. Kobayashi, H. Kohno, A. Thiaville, T. Ono, Nat. Mater., 6, 270 (2007).

[8] R. Moriya, L. Thomas, M. Hayashi, Y. B. Bazaliy, C. Rettner, S. S. P. Parkin, Nat. Phys., 4, 368 (2008). 
[9] K. Taguchi, J. -I. Ohe, G. Tatara, Phys. Rev. Lett., 109, 127204 (2012).

[10] A. Hubert, R. Schäfer, Magnetic Domains: The Analysis of Magnetic Microstructures (Springer-Verlag, Berlin Heidelberg, 2008).

[11] A. Thiaville, Y. Nakatani, J. Miltat, Y. Suzuki, Europhys. Lett., 69, 990 (2005).

[12] L. D. Geng, Y. M. Jin, J. Magn. Magn. Mater., 393, 121 (2015).

[13] A. G. Khachaturian, Theory of Structural Transformations in Solids (John Wiley \& Sons, New York, 1983).

[14] Y. M. Jin, Y. U. Wang, A. Kazaryan, Y. Wang, D. E. Laughlin, A. G. Khachaturyan, J. Appl. Phys. 92, 6172 (2002).

[15] Y. M. Jin, Acta Mater. 57, 2488 (2009).

[16] M. D. Mascaro, C. N. Nam, C. A. Ross, Appl. Phys. Lett., 96, 162501 (2010).

[17] I. Tudosa, C. Stamm, A. B. Kashuba, F. King, H. C. Siegmann, J. Stöhr, G. Ju, B. Lu, D. Weller, Nature, 428, 831 (2004).

[18] C.H. Back, R. Allenspach, W Weber, S. S. P. Parkin, D. Weller, E. L. Garwin, H. C. Siegmann, Science, 285, 864 (1999).

[19] O. Boulle, J. Kimling, P. Warnicke, M. Kläui, U. Rüdiger, G. Malinowski, H. J. M. Swagten, B. Koopmans, C. Ulysse, G. Faini, Phys. Rev. Lett., 101, 216601 (2008).

[20] L. S. E. Alvarez, K.-Y. Wang, S. Lepadatu, S. Landi, S. J. Bending, C. H. Marrows, Phys. Rev. Lett., 104, 137205 (2010).

[21] J. He, Z. Li, S. Zhang, Phys. Rev. B, 73, 184408 (2006).

[22] M. Kläui, P.-O. Jubert, R. Allenspach, A. Bischof, J. A. C. Bland, G. Faini, U. Rüdiger, C. A. F. Vaz, L. Vila, C. Vouille, Phys. Rev. Lett., 95, 026601 (2005).

[23] F. -U. Stein, L. Bocklage, M. Weigand, G. Meier, Sci. Repot, 3, 01737 (2013).

[24] S. Parkin, S. -H. Yang, Nat. Nanotechnol., 10, 195, 2015.

[25] B. Pigeau, Magnetic vortex dynamics nanostructures, Paris-Sud University, 2012. 